\title{
Housed Temperature Sensors Based on Piezoelectric Resonators for High-Temperature Applications
}

\author{
M. Schulz' ${ }^{1}$ F. Kohler ${ }^{2}$, J. Wilde ${ }^{2}$, H. Fritze ${ }^{1}$ \\ ${ }^{1}$ Institute of Energy Research and Physical Technologies, \\ Clausthal University of Technology, Am Stollen 19 B, 38640 Goslar, Germany, \\ 2 Department of Microsystems Engineering - IMTEK, \\ University of Freiburg, Georges-Köhler-Allee 103-01-105, Germany. \\ michal.schulz@tu-clausthal.de
}

\begin{abstract}
Summary:
A housed temperature sensor based on piezoelectric CTGS $\left(\mathrm{Ca}_{3} \mathrm{TaGa}_{3} \mathrm{Si}_{2} \mathrm{O}_{14}\right)$ single crystals is presented. Here, temperature data is derived from the shift of the resonance frequency, obtained from an analysis of the impedance spectra of resonators prepared from CTGS. The device is operated up to $900{ }^{\circ} \mathrm{C}$, exhibiting nearly linear temperature dependence of the resonance frequency of about $180 \mathrm{~Hz} / \mathrm{K}$ in the entire temperature range.
\end{abstract}

Keywords: piezoelectricity, temperature sensor, resonance frequency, CTGS

\section{Introduction and Objectives}

Piezoelectric devices based on high-temperature stable single crystals allow to create sensors for measurement of temperature as well as other physical properties. Thereby, the devices withstand harsh environments such as extreme temperatures or highly reducing or oxidizing atmospheres. The measured property is the resonance frequency which is governed by e.g. the temperature of the environment.

Commonly used piezoelectric materials such as quartz or lithium niobate are not suitable for hightemperature applications. Strongly increasing damping and destructive phase transitions or decomposition of the crystals limit their use up to about $\quad 400-500 \quad{ }^{\circ} \mathrm{C}$. Catangasite $\left(\mathrm{Ca}_{3} \mathrm{TaGa}_{3} \mathrm{Si}_{2} \mathrm{O}_{14}, \mathrm{CTGS}\right)$ is a commercially available member of the so-called langasite family. The crystal structure of CTGS is the same as of quartz. CTGS does not undergo any phase transitions up to its melting point at about $1350{ }^{\circ} \mathrm{C}[1]$ and exhibits an ordered crystal structure, yielding low electromechanical losses [2,3].

This paper focuses on preparation and characterization of high-temperature stable resonators based on Y-cut CTGS crystals and their integration in a gas-tight package. The temperature dependence of the resonance frequency and loss of as-prepared resonators and housed devices is compared and discussed.

\section{Experimental}

Polished Y-cut CTGS blanks with a diameter of $10 \mathrm{~mm}$ are purchased from Shanghai SICCAS
High Technology Corporation (Shanghai, China) and from FOMOS Materials (Moscow, Russia). The thickness of about $250 \mu \mathrm{m}$ is chosen for a resonance frequency of $5 \mathrm{MHz}$. Keyhole-shaped electrodes with a diameter of $5 \mathrm{~mm}$ are deposited using pulsed laser deposition (PLD) and screen printing techniques. In case of the former, a Pt/Rh alloy is deposited, as it exhibits improved stability in harsh environments [4]. In case of the latter, standard Pt paste (Ferro, 64120410) is used.

The resonators are pre-characterized in air at a ramp of $1 \mathrm{~K} / \mathrm{min}$ in temperature range from $\mathrm{RT}$ up to $850{ }^{\circ} \mathrm{C}$ and $1000^{\circ} \mathrm{C}$ for PLD and screen printed electrodes, respectively. Thereby, the impedance spectra in the vicinity of the resonance frequency is measured using an Agilent E5100A network analyzer. From this data, the peak maximum and full width at half maximum (FWHM) of the real part of admittance are calculated.

Subsequently, the resonators are mounted in a housing made of $\mathrm{Al}_{2} \mathrm{O}_{3}$ (see Fig. 1). Thereby, the CTGS is glass-soldered with a support structure. The electrical contact between connector pads and the resonator is realized using $\mathrm{Pt}$ bond wires. Finally, a cap is mounted over the resonator and sealed using glass solder technique.

The complete device is characterized in a furnace up to $900{ }^{\circ} \mathrm{C}$. Thereby, the analysis as done during the pre-characterization is repeated. The obtained frequency data and loss are 
compared with those of as-prepared Y-cut resonators.

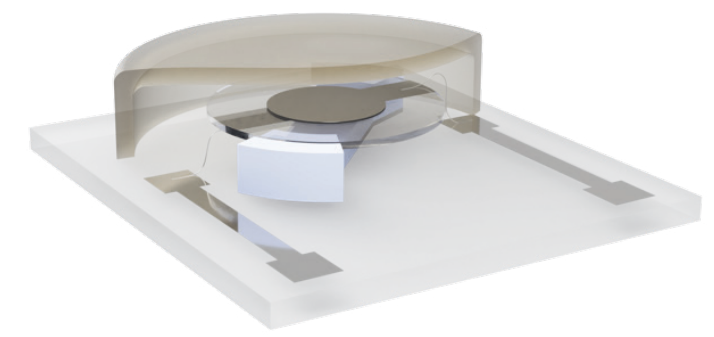

Fig. 1. Design of a temperature sensor based on CTGS.

\section{Results and Discussion}

The relative changes of resonance frequencies for as-prepared and housed resonators are shown in Fig. 2. Since the screen printed electrodes are fired in a furnace prior to the measurement, they introduce low mechanical strain to the resonator. In case of the PLD electrodes a small hysteresis of the resonance frequency is measured during first temperature ramp. The relative change of the frequency for two different crystal manufacturers is the same. The absolute data spreads by about $2 \%$ between investigated samples. This is attributed to slight differences in the thickness of as-prepared blank Y-cuts.

The loss expressed in form of inverse $Q$ factor [5] is shown in Fig. 3. Due to very narrow FWHM of the admittance peak, the calculated $Q$ value at low temperatures exhibits large noise. The increase of $Q^{-1}$ at $350-500{ }^{\circ} \mathrm{C}$ is attributed to anelastic relaxation of point defects [3]. At temperatures above $700{ }^{\circ} \mathrm{C}$ the loss is dominated by piezoelectric/conductivity relaxation [3]. As shown in Fig. 3, the loss measured for the housed device is nearly identical to those of an as-prepared SICCAS resonator. The FOMOS material exhibits significantly lower loss throughout entire temperature range.

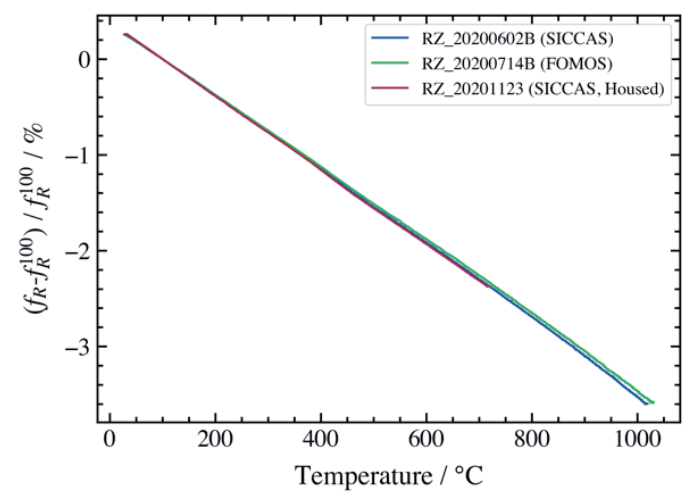

Fig. 2. Relative change of resonance frequency for CTGS manufactured by SICCAS and FOMOS as well as housed CTGS. Thereby, the housed resonator was annealed up to $700^{\circ} \mathrm{C}$ only.

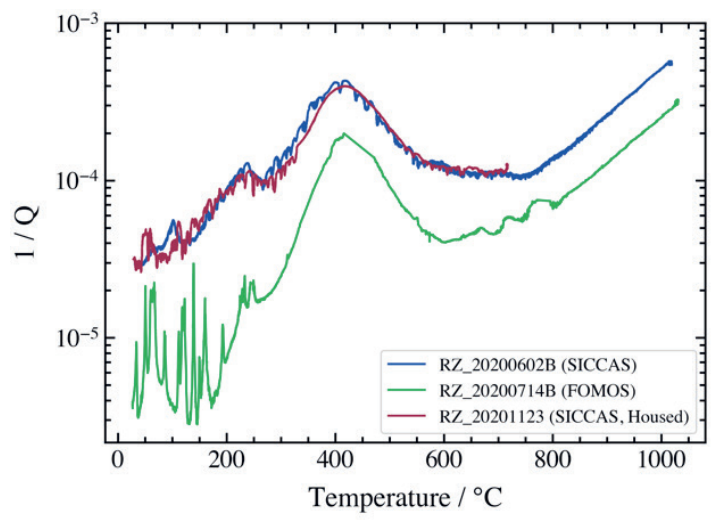

Fig. 3. Loss measured in CTGS samples and housed device as a function of temperature.

\section{Conclusions}

CTGS is a promising material for high-temperature stable sensor devices. Thanks to ordered crystal structure it exhibits low losses in comparison to some other members of langasite family. A nearly linear dependence of the resonance frequency from temperature of Y-cut CTGS simplifies the frequency to temperature conversion allowing to perform it even on low-cost microcontrollers. The housed sensor is tested up to $900{ }^{\circ} \mathrm{C}$. The impact of the housing on resonators performance is negligible.

\section{Acknowledgement}

The authors thank the German Research Foundation (Deutsche Forschungsgemeinschaft, DFG) for financial support under grants FR 1301/35-1 and WI1987/6-1.

\section{References}

[1] F. Yu, S. Zhang, X. Zhao, D. Yuan, L. Qin, Q. Wang, T. R. Shrout, Investigation of Ca3TaGa3Si2O14 piezoelectric crystals for high temperature sensors, J. Appl. Phys. 109, 114103 (2011)

[2] Yu. Suhak, M. Schulz, W. L. Johnson, A. Sotnikov, H. Schmidt, H. Fritze, Electromechanical Properties and Charge Transport of $\mathrm{Ca}_{3} \mathrm{TaGa}_{3} \mathrm{Si}_{2} \mathrm{O}_{14}$ (CTGS) Single Crystals at Elevated Temperatures, Solid State Ionics 317, 221 228 (2018)

[3] Yu. Suhak, W. L. Johnson, A. Sotnikov, H. Schmidt, and $\mathrm{H}$. Fritze, Transport and Electromechanical Properties of $\mathrm{Ca}_{3} \mathrm{TaGa}_{3} \mathrm{Si}_{2} \mathrm{O}_{14}$ Piezoelectric Crystals at Extreme Temperatures, MRS Advances 4, 515-521 (2019); doi: 10.1557/adv.2019.16

[4] D. Richter, M. Schulz, S. Sakharov, Z.J. Davis, H. Fritze, Surface Acoustic Wave Devices: Materials Stability in Harsh Environments, MRS Proceed. 1519 (2013)

[5] H. Fritze, High-Temperature piezoelelctric crystals and devices, J. Electroceramics 26, 122-161 (2011) 